\title{
Dynamic Traffic Grooming in Joint Switching (JoS)-enabled Flex-Grid/SDM Optical Core Networks
}

\author{
R. Rumipamba-Zambrano(1), J. Perelló(1), and S. Spadaro(1) \\ (1) Universitat Politècnica de Catalunya (UPC), Barcelona, Spain, e-mail: rrumipam@ac.upc.edu
}

\begin{abstract}
We propose and evaluate a dynamic traffic grooming strategy enhancing the performance of JoS-enabled Flex-Grid/SDM networks. Results disclose up to $120 \%$ and $44 \%$ additional carried network traffic vs. no grooming and existing end-to-end traffic grooming solutions, respectively.
\end{abstract}

\section{Introduction}

Flex-Grid ${ }^{1}$ and Space Division Multiplexing ${ }^{2}$ (Flex-Grid/SDM) are key technologies to cope with the rapid growth of the Internet traffic, thanks to their superior capacity and spectrum utilization flexibility. As pointed-out in the SDM literature, its near-term realization will rely on the joint switching (JoS) ${ }^{3}$ technique, switching one spectrum portion across all spatial channels at once. Using this technique, all spatial channels are treated as a single entity and, therefore, the space dimension can be considered as fixed. Meanwhile, a long-term SDM realization will introduce flexibility in both spectral and space dimensions, thus coming up with spectrally and spatially flexible optical networks requiring space and spectrum switching granularity, known as independent switching $(\operatorname{InS})^{3}$.

Flex-Grid/SDM networks realizing JoS reduce the complexity and cost of nodes by requiring a lower amount of spectrum selective switches ${ }^{4}$, forcing to allocate sub-channels in the form of spatial super-channels (Spa-SChs). Spa-SChs generated/detected by flexible transponders further reduce costs by avoiding frequency combs, sharing laser sources and facilitating the joint digital signal processing at receivers ${ }^{4}$. Alternative SCh configurations ${ }^{5}$ are spectral (Spe-SChs) and spectral-spatial superchannels (S2-SChs), commonly related to InS.

However, JoS can lead to spectrum wastage because of its spatial rigidity. As a result, the performance of JoS-enabled Flex-Grid/SDM networks can be negatively affected ${ }^{6}$. This spectrum wastage (the spatial-spectral resources left unused after the allocation of demands) can be leveraged by co-routed demands, performing end-to-end spatial traffic grooming (e2e-grooming) ${ }^{6}$. This work explores the fittest SCh configuration during the e2egrooming operation. We name this strategy as dynamic e2e-grooming and we evaluate its benefits under several traffic conditions.

\section{End-to-End Spatial Traffic Grooming}

Thanks to the spectrum flexibility given by FlexGrid, for the same demand $d$, different SCh configurations are valid to allocate it. Specifically, given a fiber with $S$ spatial channels different $\left(n_{s}, n_{f s}\right)$ tuples are possible to serve $d$, being $n_{s}$ the required number of spatial channels and $n_{f s}$ the required number of frequency slots (FSs) per spatial channel. For instance, for a demand with bit-rate $r_{d}=400 \mathrm{~Gb} / \mathrm{s}$ using Dualpolarized (DP) QPSK modulation format with Spectral Efficiency (SE) at the Nyquist limit (4 $\mathrm{b} / \mathrm{s} \cdot \mathrm{Hz}), \mathrm{S}=9$, FS width $(\mathrm{W})=12.5 \mathrm{GHz}$ and 7.5 $\mathrm{GHz}$ guard-band (GB), SCh candidates $(\Omega)$, expressed as a set of $\left(n_{s}, n_{f s}\right)$ tuples are: $\Omega=$ $\{(1,9),(2,5),(3,4),(4,3), \quad(6,2)\}$, where $n_{f s}=$ $\left\lceil\left(r_{d} /\left(n_{s} \cdot S E\right)+G B\right) / W\right\rceil$.

The typical goal in JoS-enabled FlexGrid/SDM networks is to allocate the finest spectrum portion across $n_{s}$ spatial channels in order to maximize the network Grade-ofService ${ }^{7}$. Therefore, among the SCh candidates in the previous $\Omega$ example, the last tuple $(6,2)$ would be the most suitable one, which would also be obtained following the procedure known as Partial Core (or Space) Assignment (PCA) ${ }^{7}$. Depending on the traffic profile, it has been demonstrated that JoS performance can be significantly worse than that of $\operatorname{InS}^{8}$ due to its spectrum wastage. In order to enhance it, some strategies have been proposed ${ }^{6,9}$. For example, e2e-grooming aims to reuse lightpaths for other demands having common source (s) and destination ( $\mathrm{t}$ ) nodes, always targeting a $n_{f s}$ minimization when allocating them. This procedure can be called as predefined e2egrooming and causes that some lightpaths cannot be reused because the free spatialspectral resources are not able to accommodate other demands ${ }^{6}$. To address this issue, a possibility can be to test other unexplored SCh configurations during the e2e-grooming (i.e., other tuples in $\Omega$ including Spe- or S2-SChs). If one lightpath has free spatial-spectral resources to groom another demand, we could start testing from the least demanding SCh configuration (in terms of $n_{s}$ ) to the most demanding one. This procedure can be called as dynamic e2egrooming. For example, Fig. 1 shows a Spa-

(c) 2018 IEEE. Personal use of this material is permitted. Permission from IEEE must be obtained for all other uses, in any current or future media, including reprinting/republishing this material for advertising or promotional purposes,creating new collective works, for resale or redistribution to servers or lists, or reuse of any copyrighted component of this work in other works. DOI: 10.1109/ECOC.2018.8535160 
SCh allocation for the $400 \mathrm{~Gb} / \mathrm{s}$ demand (d1), previously introduced. After allocating $d 1$ there would be $3 \times 2$ free spatial-spectral resources that might be used to allocate a co-routed demand, e.g., d2 of $100 \mathrm{~Gb} / \mathrm{s}$ with DP-QPSK. The SCh candidates $(\Omega)$ of $d 2$ are: $(1,3),(2,2)$ and $(5,1)$. The predefined e2e-grooming would select the $(5,1)$ tuple, which does not suitably fit into the free spatial-spectral resources. Nevertheless, following our dynamic e2e-grooming proposal, the first candidate tuple $(1,3)$ is discarded because it requires higher spectral resources, whereas the second one $(2,2)$ fits properly and even 1x2 spatial-spectral resources are still available to groom other demand in $\mathrm{S} 9$.

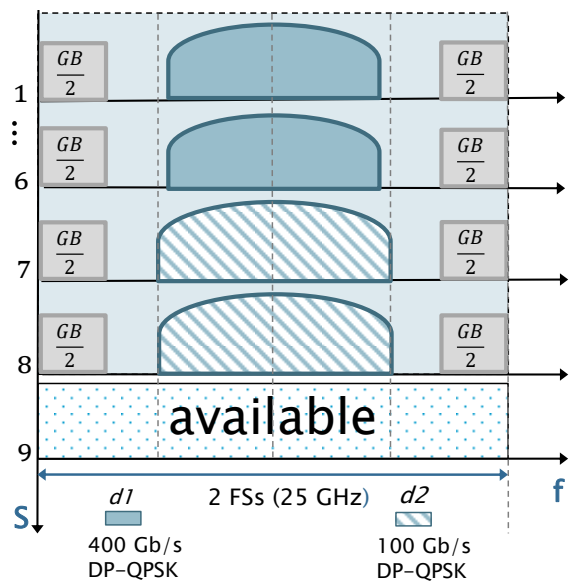

Fig. 1: Dynamic e2e-grooming for two co-routed demands

\section{RMSSA heuristic}

In this section, we elaborate on the Route, Modulation Format, Space and Spectrum Assignment (RMSSA) greedy heuristic for JoSenabled Flex-Grid/SDM networks with dynamic e2e-grooming. In general, when a new demand $d$ arrives at the network, it should be firstly checked if the e2e-grooming operation is feasible. To this end, all lightpaths sharing the same $s$ and $t$ nodes as $d$ are found. Then, for each of these lightpaths the most spectrally efficient (MSE) modulation format is selected according to its physical length, in order to compute $\Omega$ from $n_{s}=1$ to $n_{s}=S$. $i$-th tuples $\left(n_{s}, n_{f s}\right)$ that do not yield lower $n_{f s}$ than their $(i$ 1)-th are trivially discarded at the very beginning. The available spatial-spectral resources per lightpath are contrasted with those required by each SCh candidate $\in \Omega$. If one SCh candidate suitably fits into the available resources, $d$ is considered as served; otherwise, a new lightpath has to be established. For this purpose, the $k=3$ shortest paths are used as candidate physical paths. Starting with the shortest one, the following procedure is executed: 1) Select the MSE modulation format with an optical reach equal or higher than the physical path length; 2) Compute the $\left(n_{s}, n_{f s}\right)$ tuple by using the PCA strategy, i.e., the SCh configuration minimizing $\left.n_{f s} ; 3\right)$ Find if continuous and contiguous $n_{f s}$ FSs are available in the spectrum of the first spatial channel along the candidate path. If so, directly reserve them across $n_{s}$ spatial channels and consider $d$ as served. Otherwise, subsequent candidate paths are analysed. If no candidate path can allocate $d$, it is finally considered as blocked.

\section{Simulation Results and Discussion}

To evaluate the proposed dynamic e2egrooming, we consider the DT12 National backbone network ${ }^{2}$ with 12 nodes and 40 unidirectional fiber links (average link length 243 $\mathrm{km})$. Each fiber link consists of 22 spatial channels (bundle of standard single-mode fibers -SSMF-), each one with $320 \mathrm{FSs}$ of $12.5 \mathrm{GHz}$ (4 THz C-band). The optical reach estimation per Spa-SCh is computed by following the procedure detailed in ref. ${ }^{7}$ for SSMF with up to 32 GBaud DP-BPSK, DP-QPSK, DP-16QAM, DP-64QAM signals.

A dynamic scenario where demands arrive at the network following a negative exponentially distributed inter-arrival time (IAT) is assumed. Each request asks for a bidirectional lightpath between uniformly distributed $\mathrm{s}$ and $\mathrm{t}$ nodes with bit-rate $r_{d}$ during a certain holding time $(\mathrm{HT})$, also following a negative exponential distribution. The $r_{d}$ value follows several traffic profiles (TPs) with different mean $(\mu)$ and standard deviation $(\sigma)$ bit-rate. Different offered loads $(L)$ are obtained by fixing the IAT and varying the $H T$ accordingly ( $L=H T / I A T)$. To get statistically relevant results, we offer $5 \times 10^{5}$ bidirectional requests per execution.

Fig. 2(a-f) show the Bandwidth Blocking Probability (BBP) vs. L (in Pb/s) for 6 TPs $(\mu, \sigma)$ from TP1 to TP6, respectively. Three curves are shown per graph disclosing results without e2egrooming (No-Grooming, plots in purple), with predefined e2e-grooming (Predef-Grooming, plots in blue) and with dynamic e2e-grooming (Dyna-Grooming, plots in red). In the first set of simulations -Fig. 2(a-c) on top- the $\mu$ value per connection is the same, while the $\sigma$ one is increased, namely, TP1 $(2.0,0.0)$, TP2 $(2.0,0.8)$ and TP3 $(2.0,1.6)$, where $\mu$ and $\sigma$ values are in $\mathrm{Tb} / \mathrm{s}$. Conversely, in the second set of simulations -Fig. 2(d-f) at the bottom- the $\sigma$ value is the same, while the $\mu$ one is increased, namely, TP4 $(0.5,0.4)$, TP5 $(1.0,0.4)$ and TP6 $(2 \cdot 0,0.4)$. 

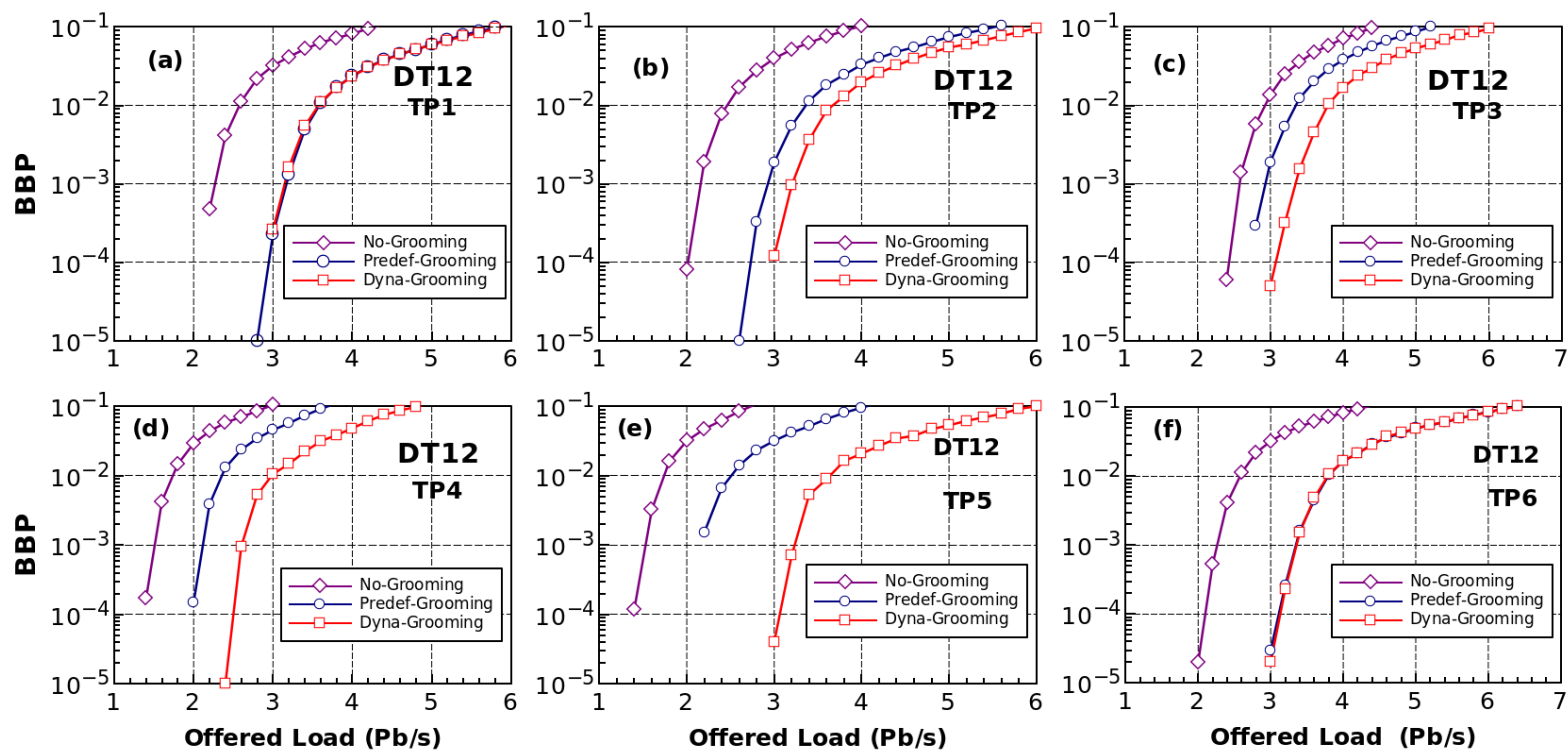

Fig. 2: BBP vs. Offered Load (in $\mathrm{Pb} / \mathrm{s}$ ) for different TPs, (top): fixed $\mu$ and increasing $\sigma$, (bottom): fixed $\sigma$ and increasing $\mu$

Looking at the results, for a fixed $\mu=2$, the higher the $\sigma$, the higher the benefits (in terms of $\mathrm{L})$ of dynamic versus predefined e2e-grooming. Specifically, for a homogeneous TP1 (i.e., $\sigma=0$ ) the benefits of dynamic e2e-grooming are inappreciable. However, for heterogeneous TPs (i.e., $\sigma>0$ ) these benefits are up to $6 \%$ and $10 \%$ for $\sigma=0.8-\mathrm{TP} 2-$ and $\sigma=1.6-\mathrm{TP} 3-$, respectively, for a $1 \%$ target BBP. Meanwhile, for a 10\% BBP these benefits raise up to $7 \%$ and $15 \%$ for both cases of $\sigma=0.8$ and $\sigma=1.6$, respectively.

Regarding the second set of simulations, fixing $\sigma=0.4$ and varying $\mu$, dynamic e2egrooming benefits can raise up to $25 \%$ and $44 \%$ for TP4 and TP5, respectively, for $1 \%$ target BBP. Moreover, Fig. 2(d-f) show the impact of the size of demands (in terms of bit-rate). As observed, the higher the bit-rate of demands, the higher the supported $L$ (therefore, the network throughput) under the three strategies (no, predefined and dynamic e2e-grooming). For instance, for a $1 \%$ target BBP, $\mathrm{L}$ can increase up to $27 \%, 58 \%$ and $47 \%$ for no grooming, predefined and dynamic e2e-grooming, respectively, when $\mu$ value per TP ranges from $0.5 \mathrm{~Tb} / \mathrm{s}-$ Fig. 2(d)- to $2 \mathrm{~Tb} / \mathrm{s}$-Fig. 2(f)-. Meanwhile, for a $10 \%$ BBP the same percentages raise up to $40 \%, 86 \%$ and $35 \%$, respectively. Among the three strategies, the results show that predefined e2e-grooming is the most sensible to the size of demands.

Taking into account the joint impact of $\mu$ and $\sigma$ values, the benefits of e2e-grooming are more significant for low/moderate bit-rate and heterogeneous demands, as observed for TP4 and TP5 -Fig. 2(d,e)-, whereas these benefits are reduced for high bit-rate and very heterogeneous demands, such as the case of TP3 -Fig. 2(c)-. Specifically, for a 1\% target BBP, for TP4 and TP5, the benefits of e2egrooming are up to $50 \%$ for the predefined strategy, against an up to $120 \%$ for the dynamic one. Meanwhile, for TP3, the benefits are reduced by $15 \%$ and $27 \%$, for predefined and dynamic e2e-grooming, respectively. Finally, according to the results, dynamic e2e-grooming does not yield benefits for high bit-rates and homogeneous or quasi-homogeneous TPs, as observed for TP1 and TP6.

\section{Conclusions}

Dynamic e2e-grooming strategy in JoS-enabled Flex-Grid/SDM networks yields high benefits compared to predefined e2e-grooming especially under moderate traffic profiles in terms of bit-rate and heterogeneity. Particularly, a performance enhancement of up to $44 \%$ has been showed. Future works can address complexity analysis related to control plane and transponders for dynamic e2e-grooming.

\section{Acknowledgements}

This work has been funded by the Spanish National Project (TEC2017-90034-C2-1-R and TEC201790034-C2-2-R) under FEDER contribution and by SENESCYT - Ecuador.

\section{References}

[1] O. Gerstel et al., IEEE Commun. Mag. 50, S12, 2012

[2] P. Winzer, Nat. Photonics 8, 345, 2014.

[3] D. M. Marom et al., IEEE Commun. Mag. 53, 60, 2015

[4] J. M. Rivas Moscoso et al., in ECOC 2016, M.1.F.4

[5] D. Klonidis et al., IEEE Commun. Mag. 53, 69, 2015

[6] R. Rumipamba-Zambrano et al., in OFC 2017, W4F.8

[7] R. Rumipamba-Zambrano et al., Opt. Switch. Net. 27, 93, 2018

[8] B. Shariati et al., in ECOC 2016, M.1.F.1

[9] F. Pederzolli et al., J. Opt. Comm. Netw. 9, B1, 2017 